\title{
Hiperplasia Neo-Intimal Intra-Stent e Progressão de Placa Aterosclerótica no Vaso-Alvo em Pacientes Tratados com Stents Liberadores de Zotarolimus
}

Andre F. L. Brito ${ }^{1}$, José de Ribamar Costa Jr. ${ }^{1}$, Alaor Mendes C. Jr. ${ }^{1}$, Julio P. Maia' ${ }^{1}$, Fausto Feres ${ }^{1}$, Alexandre Abizaid ${ }^{1}$, Ricardo A. Costa ${ }^{1}$, Rodolfo Staico ${ }^{1}$, Dimytri Siqueira ${ }^{1}$, Luiz Alberto Mattos ${ }^{1}$, Amanda G. M. R. Sousa' ${ }^{1}$ J. Eduardo Sousa ${ }^{1}$

\section{RESUMO}

Fundamentos: Após intervenções coronárias percutâneas, tem sido descrita uma reação inflamatória local que está diretamente relacionada à hiperplasia neo-intimal e à reestenose. O quanto esse processo inflamatório pode levar à progressão de placa aterosclerótica no vaso tratado ou nas outras artérias coronárias é ainda motivo de debate. Objetivo: Avaliar se há relação entre o grau de hiperplasia intimal intra-stent e a progressão de placa no vaso tratado. Método: Estudo retrospectivo com 36 pacientes com 40 lesões tratados com stents liberadores de zotarolimus, avaliados com ultra-sonografia intracoronária (USIC) após o procedimento e no reestudo protocolar tardio. Obtidos o porcentual do volume de obstrução intimal intra-stent $(\% \vee O)$ e a mudança de volume da placa aterosclerótica fora do segmento tratado ( $\triangle \mathrm{VAT})$. Avaliamos a intensidade da relação entre as variáveis \% VO e $\triangle \mathrm{VAT}$ por meio do coeficiente de correlação de Spearman. Resultados: O \% VO foi de $14,5 \% \pm 14,9 \%$. Observada progressão de placa nas porções proximais $\left(20,04 \mathrm{~mm}^{3} / \mathrm{mm}^{2} \pm 58,61 \mathrm{~mm}^{3} / \mathrm{mm}\right)$ e distais $\left(7,37 \mathrm{~mm}^{3} / \mathrm{mm} \pm 25,86 \mathrm{~mm}^{3} / \mathrm{mm}\right)$ ao segmento tratado com stent. Não houve correlação entre o porcentual de hiperplasia neo-intimal e o grau de progressão de placa proximal $(-0,015 ; p=0,93)$, distal $(-0,166 ; p=0,36)$ ou em ambos os segmentos $(-0,026 ; p=0,89)$. Conclusão: Neste grupo de pacientes tratados com stents liberadores de zotarolimus, a ausência de correlação entre o volume de obstrução intimal intra-stent e a progressão de placa fora do segmento tratado sugere a existência de diferentes mecanismos inflamatórios por trás desses processos.

DESCRITORES: Stents farmacológicos. Reestenose coronária. Ultra-sonografia. Aterosclerose.

\footnotetext{
Instituto Dante Pazzanese de Cardiologia - São Paulo, SP. Correspondência: José de Ribamar Costa Jr. Av. Dr. Dante Pazzanese, 500 - Ibirapuera - São Paulo, SP - CEP 04012-909

E-mail: rmvcosta@uol.com.br

Recebido em: 4/8/2008 • Aceito em: 12/12/2008
}

\section{SUMMARY}

Intra-Stent Neointimal Hyperplasia and Plaque

Progression in the Target Vessel in Patients Treated with Zotarolimus Eluting Stents

Background: A local inflammatory response has been described after percutaneous coronary intervention and has been associated with neointimal hyperplasia and restenosis. Whether this inflammatory process might cause atherosclerotic progression in the same coronary artery or in the other coronary arteries is a matter of debate. Objectives: To assess a possible relation between the degree of neointimal hyperplasia and the progression of atherosclerotic plaque in the nearby stented segments. Methods: Retrospective study in which 36 patients with 40 lesions treated with zotarolimus eluting stents were studied with intravascular ultrasound (IVUS) during the index and follow-up procedures. In-stent percent volume obstruction $(\% \mathrm{VO})$ and change in atheroma volume in the arterial subsegments outside the stented segment $(\triangle \mathrm{ATV})$ were obtained. We assessed the strength of the relationship between \%VO and $\triangle$ ATV by the Spearman correlation coefficient. Results: $\%$ VO was $14.5 \% \pm 14.9 \%$. Plaque progression was observed in proximal $\left(20.04 \mathrm{~mm}^{3} / \mathrm{mm}^{2} \pm 58.61 \mathrm{~mm} 3 / \mathrm{mm}\right)$ and distal $\left(7.37 \mathrm{~mm}^{3} / \mathrm{mm} \pm 25.86 \mathrm{~mm}^{3} / \mathrm{mm}\right)$ arterial subsegments nearby the stented segment. There was no correlation between the degree of neointimal hyperplasia and the degree of plaque progression either in the proximal $(-0.015 ; p=0.93)$, distal $(-0.166 ; p=0.36)$ or both segments $(-0.026, p=0.89)$. Conclusion: In this group of patients treated with zotarolimus eluting stents, the lack of correlation between the degree of neointimal hyperplasia and plaque progression suggests different inflammatory reactions behind those phenomena.

DESCRIPTORS: Drug-eluting stents. Coronary restenosis. Ultrasonography. Atherosclerosis.
$\mathrm{T}$ anto a progressão de doença aterosclerótica como a reestenose intra-stent após angioplastia transluminal percutânea coronária (ATC) fazem parte do espectro de manifestações da doença coronária, cuja etiopatogenia parece estar intimamente relacionada a processos inflamatórios locais e sistêmicos ${ }^{1-4}$. Embora a 
progressão de placa aterosclerótica e a reestenose intrastent tenham em comum esse processo inflamatório, sua associação ainda não foi claramente demonstrada ${ }^{5,6}$. O presente estudo teve como objetivo investigar o comportamento de vasos tratados com stent farmacológico liberador de zotarolimus (Endeavor ${ }^{\mathrm{TM}}$, Medtronic Vascular, Santa Rosa, CA, Estados Unidos), avaliando-se se há relação entre grau de hiperplasia intimal intra-stent e progressão do ateroma fora do segmento de vaso tratado, por meio de ultra-sonografia intracoronária (USIC) seriada.

\section{MÉTODO}

\section{População avaliada}

Estudo retrospectivo, constituindo-se de subanálise de pacientes pertencentes ao registro multicêntrico Endeavor 5 (E5), que incluiu pacientes de moderada complexidade clínico-angiográfica.

Foram incluídos nesta análise pacientes tratados no período entre janeiro e março de 2006, que apresentavam lesões coronárias de novo em artérias coronárias nativas, abordadas percutaneamente com implante de stents Endeavor ${ }^{\mathrm{TM}}$, nos quais foi realizado estudo ultra-sonográfico imediatamente após o implante do stent e no seguimento angiográfico de seis meses. De acordo com os critérios do Registro E5, foram excluídos pacientes tratados com angioplastia primária ou de resgate na fase aguda do infarto agudo do miocárdio (IAM) ou com contra-indicação para terapia farmacológica antiplaquetária dupla.

Dentre os 100 pacientes incluídos no E5 em nossa Instituição, 64 foram excluídos desta análise pelos seguintes motivos:

- 44 pacientes por possuírem somente uma avaliação ultra-sonográfica (índex ou reestudo);

- 14 pacientes por não possuírem segmentos pareados para análise;

- 6 pacientes por dificuldades técnicas para análise.

Todos os pacientes assinaram termo de consentimento e o protocolo do estudo foi aprovado pelo Comitê de Ética de nossa Instituição.

\section{Intervenção percutânea}

Todas as intervenções foram realizadas de acordo com os protocolos vigentes e a estratégia final de implante foi deixada a critério do operador, incluindo a decisão por realizar pré e pós-dilatação. O stent Endeavor $^{\mathrm{TM}}$ estava disponível nos seguintes tamanhos: $8 \mathrm{~mm}, 12 \mathrm{~mm}, 14 \mathrm{~mm}, 18 \mathrm{~mm}, 24 \mathrm{~mm}$ e $30 \mathrm{~mm}$ em extensão para os diâmetros de $2,25 \mathrm{~mm}, 2,5 \mathrm{~mm}$ e 2,75 mm; e nas extensões de $9 \mathrm{~mm}, 12 \mathrm{~mm}, 15 \mathrm{~mm}$, $18 \mathrm{~mm}, 24 \mathrm{~mm}$ e $30 \mathrm{~mm}$ para os diâmetros de 3,0 mm, $3,5 \mathrm{~mm}$ e 4,0 $\mathrm{mm}$. Implante de mais de um stent Endeavor $^{\mathrm{TM}}$ era permitido pelo protocolo.
A terapia antiplaquetária dupla incluiu ácido acetilsalicílico (AAS) (dose de ataque de 500 mg 24 horas antes do procedimento e manutenção de $100 \mathrm{mg} / \mathrm{dia}$ ) e clopidogrel (dose de ataque de 300 mg 24 horas antes do procedimento e manutenção de $75 \mathrm{mg} / \mathrm{dia})$. A ticlopidina $500 \mathrm{mg} / \mathrm{dia}$, iniciada três dias antes do procedimento, poderia ser utilizada para substituir o clopidogrel. Após a intervenção, AAS foi prescrito indefinidamente e o tienopiridínico, mantido por pelo menos três meses. Durante o procedimento, heparina EV foi administrada na dose de 70-100 U/kg, a fim de manter o tempo de coagulação ativada (TCA) $>250^{\prime}$ (> 200' se o paciente estivesse em uso de inibidor da glicoproteína IIb/IIla).

Eletrocardiograma foi obtido antes, imediatamente após a intervenção e na alta hospitalar. Exames laboratoriais, incluindo dosagem de CK-MB, foram realizados 24 horas antes da intervenção e repetidos 18 a 24 horas após. Em caso de alteração enzimática, foi realizada curva de CK-MB, a cada oito horas, até a normalização da mesma.

\section{Ultra-sonografia intracoronária}

A aquisição das imagens de USIC (pós-procedimento e aos seis meses de evolução) foi realizada utilizando-se um transdutor de elemento único, rotacional, com freqüência de $40 \mathrm{MHz}$, envolto numa bainha com perfil de 2,6 F, com recuo motorizado em sistema de tração automática à velocidade de $0,5 \mathrm{~mm} / \mathrm{s}$, e scanners comerciais (Galaxy 2, Boston Scientific Corp., Nattick, Massachusetts, Estados Unidos). As imagens foram digitalizadas para análise quantitativa off-line, de acordo com os critérios do American College of Cardiology's Clinical Expert Consensus Document on IVUS ${ }^{7}$.

O segmento de vaso tratado foi definido como a extensão do stent acrescida dos $5 \mathrm{~mm}$ proximais e distais (bordas do stent). A progressão da placa aterosclerótica foi avaliada fora desse segmento tratado (Figura 1).

Foram considerados neste estudo somente os pacientes que tinham os mesmos segmentos avaliados pela USIC imediatamente após a intervenção e na avaliação angiográfica protocolar de seis meses. Para realização da análise volumétrica, foi realizada a reconstrução tridimensional das imagens da USIC obtidas a cada $1 \mathrm{~mm}$, utilizando um programa de planimetria computadorizada comercialmente disponível (EchoPlaque 2, INDEC Systems Inc., Mountain View, Califórnia, Estados Unidos). As áreas do stent, luz e hiperplasia intimal (stent-luz) foram medidas dentro do stent e os volumes, calculados pela Regra de Simpson. O porcentual do volume de obstrução intimal intra-stent (\%VO) foi calculado pela razão entre o volume de hiperplasia intimal e o volume do stent x 100 .

As áreas da membrana elástica externa (MEE), luz e placa aterosclerótica (MEE-luz) foram obtidas fora do 
Brito AFL, et al. Hiperplasia Neo-Intimal Intra-Stent e Progressão de Placa Aterosclerótica no Vaso-Alvo em Pacientes Tratados com Stents Liberadores de Zotarolimus. Rev Bras Cardiol Invas. 2008;16(4):450-455.

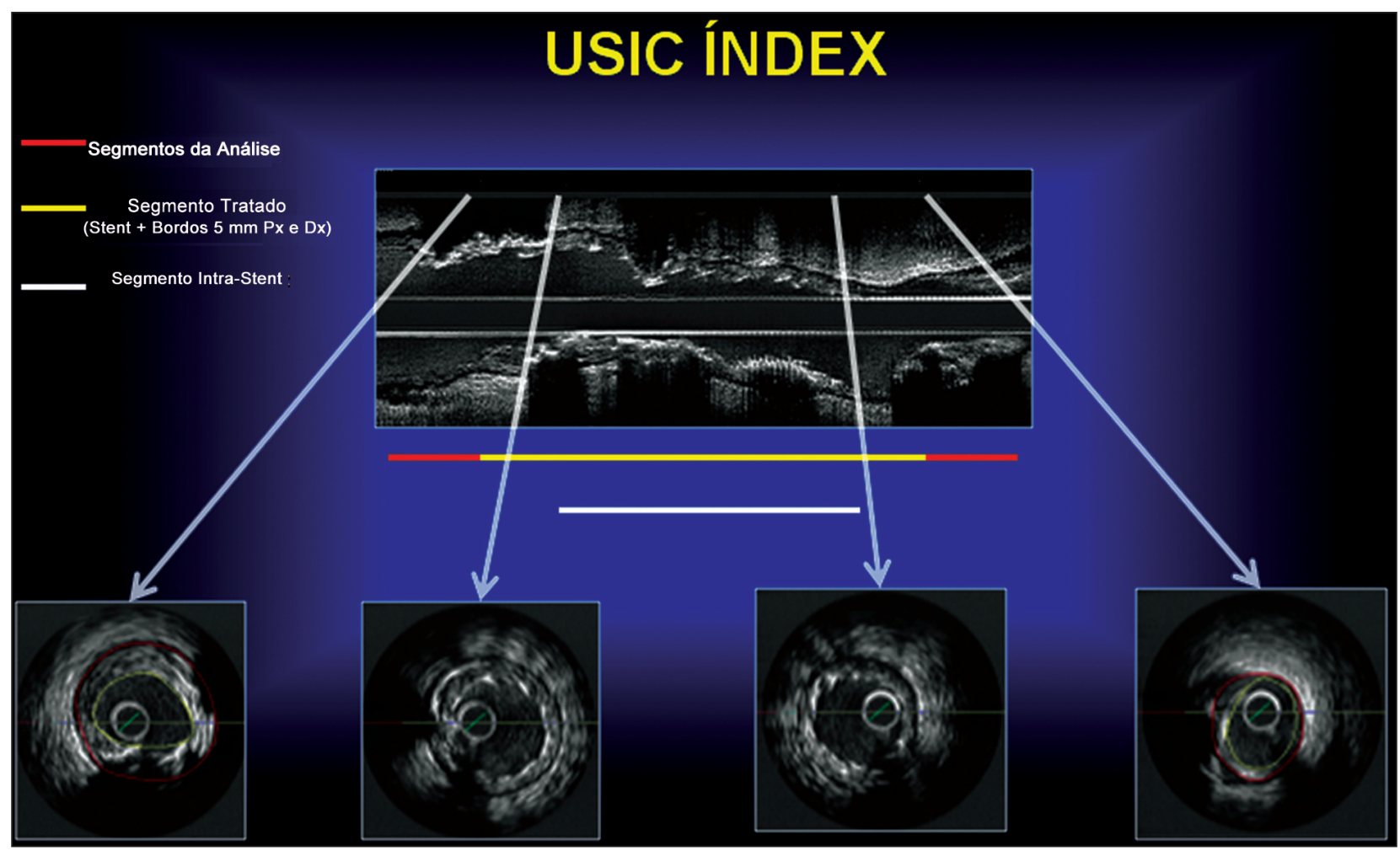

Figura 1 - Método de seleção dos segmentos analisados: traçado cinza, região onde se encontra o stent; traçado amarelo, stent + bordos 5 mm proximal e distal; e traçado vermelho, segmentos submetidos a análise sobre progressão de placa ateromatosa no presente estudo. Dx = distal; $\mathrm{PX}=$ proximal; USIC = ultra-sonografia intracoronária.

segmento tratado (stent $\pm 5 \mathrm{~mm}$ ). O volume da placa aterosclerótica (VAT) foi obtido pela soma das áreas obtidas a cada mm no segmento analisado. A mudança de VAT ( $\triangle$ VAT) foi calculada como: VAT tardio VAT procedimento. ${ }^{8-10} \mathrm{Um}$ índice de $\triangle$ VAT foi criado para permitir a comparação entre os pacientes por meio da razão $\Delta$ VAT/extensão em $\mathrm{mm}$ dos segmentos (proximal e/ou distal), visto possuírem tais segmentos comprimentos distintos entre os diferentes pacientes.

\section{Análise estatística}

As variáveis categóricas foram descritas como freqüências e as variáveis contínuas, como médias e desvio padrão.

Após testar as variáveis principais para normalidade utilizando o teste de Kolmogorov-Smirnov, comparou-se o \% VO a progressão de placa nos segmentos fora da região tratada (stent + bordos) por meio do coeficiente de correlação de Spearman. A comparação entre progressão de placa proximal versus distal foi realizada por meio do teste de Wilcoxon.

Valor de $p<0,05$ foi considerado significante do ponto de vista estatístico. As análises foram realizadas por meio do programa SPSS 12.0.

\section{RESULTADOS}

Entre os 36 pacientes incluídos nesta análise, 69,5\% eram do sexo masculino, com média de idade de 60 anos \pm 10 anos, e $25 \%$ eram diabéticos. A artéria descendente anterior foi a mais freqüentemente tratada (40\%). A Tabela 1 apresenta as principais características clínicas dos pacientes incluídos. O tempo médio de seguimento angiográfico foi de 203 dias \pm 33,7 dias.

A Tabela 2 apresenta as principais características angiográficas, com $41,2 \%$ das lesões sendo do tipo B/C (de acordo com a definição do American College of Cardiology/American Heart Association - ACC/AHA) e extensão média de 10,2 mm $\pm 7,8 \mathrm{~mm}$, tendo sido utilizados 46 stents para tratar 40 lesões (1,12 stent/lesão).

No que se refere à presente análise, no total, 69 segmentos (dentre os 80 possíveis) foram avaliados, sendo 37 proximais e 32 distais. A Tabela 3 mostra as principais características ultra-sonográficas. O \% VO na avaliação angiográfica protocolar de seis meses foi de $14,5 \% \pm 14,9 \%$. O grau de progressão de placa proximal foi de $20,04 \mathrm{~mm}^{3} / \mathrm{mm}^{2} \pm 58,61 \mathrm{~mm}^{3} / \mathrm{mm}^{2}$ e de placa distal foi de $7,37 \mathrm{~mm}^{3} / \mathrm{mm} \pm 25,86 \mathrm{~mm}^{3} / \mathrm{mm}$. Na análise combinada (proximal + distal), houve progressão de $29,14 \mathrm{~mm}^{3} / \mathrm{mm} \pm 73,02 \mathrm{~mm}^{3} / \mathrm{mm}$ nos segmentos avaliados. 
TABELA 1

Características clínicas basais dos pacientes

\begin{tabular}{lc}
\hline Características & Número de pacientes (n = 36) \\
\hline Idade (anos) & $60 \pm 10$ \\
Sexo masculino & $25(69,5 \%)$ \\
Hipertensão & $28(78,8 \%)$ \\
Diabetes melito & $9(25 \%)$ \\
Tabagismo & $6(16,7 \%)$ \\
Dislipidemia & $21(58,3 \%)$ \\
História familiar & $5(13,8 \%)$ \\
Insuficiência renal crônica & $3(8,3 \%)$ \\
Infarto do miocárdio prévio & $9(25 \%)$ \\
ATC prévia & $6(16,7 \%)$ \\
Revascularização do & - \\
miocárdio prévia & \\
Tempo de seguimento (dias) & $203 \pm 33,7$ \\
Medicações (inicial) & \\
$\quad$ AAS & $36(100 \%)$ \\
Betabloqueadores & $36(100 \%)$ \\
IECA/BRA** & $32(90 \%)$ \\
Estatinas & $36(100 \%)$ \\
Clopidogrel & $36(100 \%)$ \\
Perfil lipídico inicial & $173 \pm 108$ \\
LDL-colesterol (mg/dl) & $98 \pm 31$ \\
HDL-colesterol (mg/dl) & $43 \pm 13$ \\
Triglicérides (mg/dl) & \\
\hline
\end{tabular}

* Em outros vasos.

AAS = ácido acetilsalicílico; ATC = angioplastia transluminal percutânea coronária; $\mathrm{BRA}=$ bloqueadores de receptores de angiotensina; HDL-colesterol = colesterol de lipoproteína de alta densidade; IECA = inibidores da enzima conversora da angiotensina; LDL-colesterol = colesterol de lipoproteína de baixa densidade; $\mathrm{n}=$ número de pacientes.

Não houve correlação entre o \% $\% \mathrm{O}$ e a progressão de placa em quaisquer segmentos (Figuras 2 a 4): segmento proximal, correlação de Spearman de -0,015 $(p=0,93)$; segmento distal: correlação de Spearman de $-0,166$ ( $p=0,36)$; e ambos os segmentos (proximal + distal), correlação de Spearman de -0,026 ( $p=0,89)$. Também não se observou diferença estatística significativa entre o grau de progressão de placa no segmento proximal versus distal $(p=0,36)$.

\section{DISCUSSÃo}

O presente estudo, até onde é de nosso conhecimento, representa a primeira avaliação entre a progressão neo-intimal no interior de um stent farmacológico e a progressão de placa fora dos segmentos tratados. Como principal achado, destacamos a inexistência de correlação entre esses dois fenômenos nessa população tratada com o stent Endeavor ${ }^{\mathrm{TM}}$.
TABELA 2

Características angiográficas

\begin{tabular}{lc}
\hline Características & $\mathbf{3 6}$ pacientes/40 lesões \\
\hline Artéria tratada & \\
Artéria descendente anterior & $16 / 40 \%$ \\
Artéria circunflexa & $(9) 22,5 \%$ \\
Artéria coronária direita & $(10) 25 \%$ \\
Ramo diagonal & $(3) 7,5 \%$ \\
Ramo marginal & $(1) 2,5 \%$ \\
Tipo de lesão* & \\
De novo & $100 \%$ \\
A & $58,8 \%$ \\
B & $17,6 \%$ \\
C & $23,6 \%$ \\
Extensão da lesão (mm) & $10,2 \pm 7,8$ \\
Diâmetro dos stents (mm) & $3,03 \pm 0,37$ \\
Comprimento dos stents (mm) & $17,9 \pm 5,93$ \\
Lesão por paciente & 1,12 \\
Extensão do stent/extensão da & 1,5 \\
lesão (mm) & \\
Número de stents/número de & $46 / 40(1,12)$ \\
lesões (stent por lesão) & \\
\hline * Conforme classificação do American College of Cardiology/ \\
American Heart Association. \\
\hline
\end{tabular}

Ambas as situações têm explicações fisiopatológicas diferentes ${ }^{11-13}$. A doença aterosclerótica parece ser desencadeada pelo contato entre as partículas de colesterol de lipoproteína de baixa densidade (LDL-colesterol) oxidado e o endotélio; a disfunção endotelial resultante ativa plaquetas, macrófagos e linfócitos, promovendo migração de células musculares lisas e culminando no surgimento e na progressão da placa aterosclerótica e, por vezes, sua instabilização ao longo do tempo ${ }^{1,2,11,12}$. Esse processo usualmente tem evolução cronológica lenta e está diretamente correlacionado com os níveis lipídicos séricos.

Por outro lado, o processo inflamatório secundário ao implante do stent, ainda que apresente os mesmos componentes celulares inflamatórios que a doença aterosclerótica (monócitos/macrófagos, linfócitos, plaquetas ativadas, migração de células musculares lisas e deposição de colágeno), não apresenta evolução tão lenta, nem depende dos níveis lipídicos para ocorrer ${ }^{11-15}$, estando diretamente relacionado à injúria vascular causada pela insuflação do balão e implante do stent e associado a uma resposta vascular idiossincrática.

É importante ressaltar que a evolução cronológica desse processo depende do instrumental utilizado na abordagem percutânea. Por exemplo, o processo inflamatório decorrente da angioplastia com cateterbalão tem seu pico durante o procedimento e nas 
TABELA 3

Características da ultra-sonografia intracoronária

\begin{tabular}{|c|c|}
\hline Características & 36 pacientes/40 lesões \\
\hline $\begin{array}{l}\text { Segmentos proximais } \\
\text { avaliados }(\%)\end{array}$ & $37 / 40(92,5)$ \\
\hline $\begin{array}{l}\text { Segmentos distais } \\
\text { avaliados (\%) }\end{array}$ & $32 / 40(80)$ \\
\hline $\begin{array}{l}\text { Total de segmentos } \\
\text { avaliados (\%) }\end{array}$ & $69 / 80(86,25)$ \\
\hline $\begin{array}{l}\text { Comprimento médio dos } \\
\text { segmentos proximais }(\mathrm{mm})\end{array}$ & $5,05 \pm 2,18$ \\
\hline $\begin{array}{l}\text { Comprimento médio dos } \\
\text { segmentos distais }(\mathrm{mm})\end{array}$ & $7,99 \pm 3,45$ \\
\hline $\begin{array}{l}\text { Comprimento total dos } \\
\text { segmentos }(\mathrm{mm})\end{array}$ & $11,06 \pm 4,74$ \\
\hline$\% \vee O$ & $14,5 \pm 14,9$ \\
\hline $\begin{array}{l}\text { Grau de progressão de } \\
\text { placa proximal }\left(\mathrm{mm}^{3} / \mathrm{mm}\right)\end{array}$ & $20,04 \pm 58,61$ \\
\hline $\begin{array}{l}\text { Grau de progressão de } \\
\text { placa distal }\left(\mathrm{mm}^{3} / \mathrm{mm}\right)\end{array}$ & $7,37 \pm 25,86$ \\
\hline $\begin{array}{l}\text { Grau de progressão de } \\
\text { placa global (mm³/mm) }\end{array}$ & $29,14 \pm 73,02$ \\
\hline
\end{tabular}

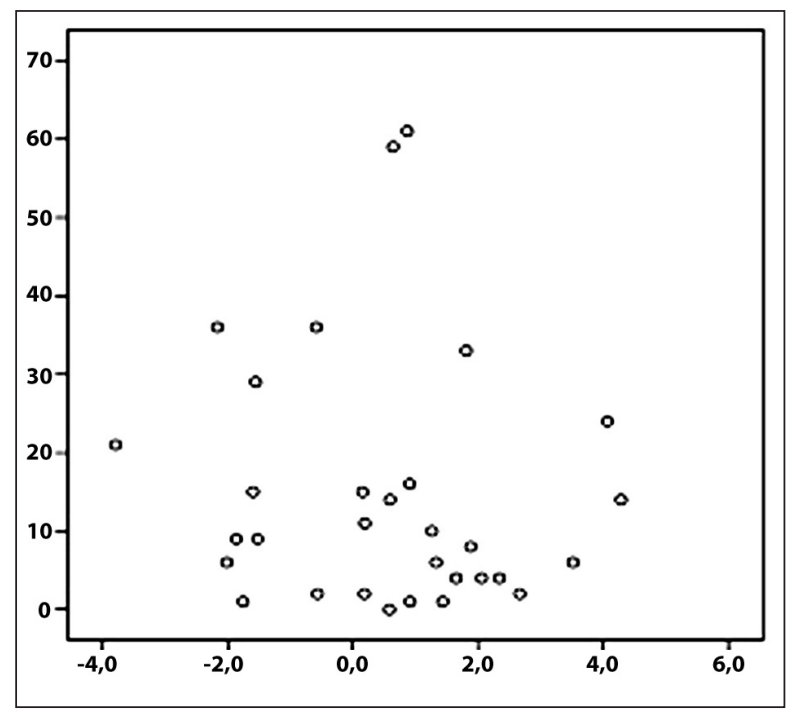

Figura 2 - Correlação entre hiperplasia neo-intimal e progressão de placa nos segmentos proximais. Correlação de Spearman de - 0,015 , $p=0,93$.

primeiras horas após sua realização. Já a introdução das endopróteses metálicas retardou essa resposta. O implante de stents não-farmacológicos induz resposta inflamatória e processo de reparação/cicatrização que se prolonga até o sexto/oitavo meses da interven-

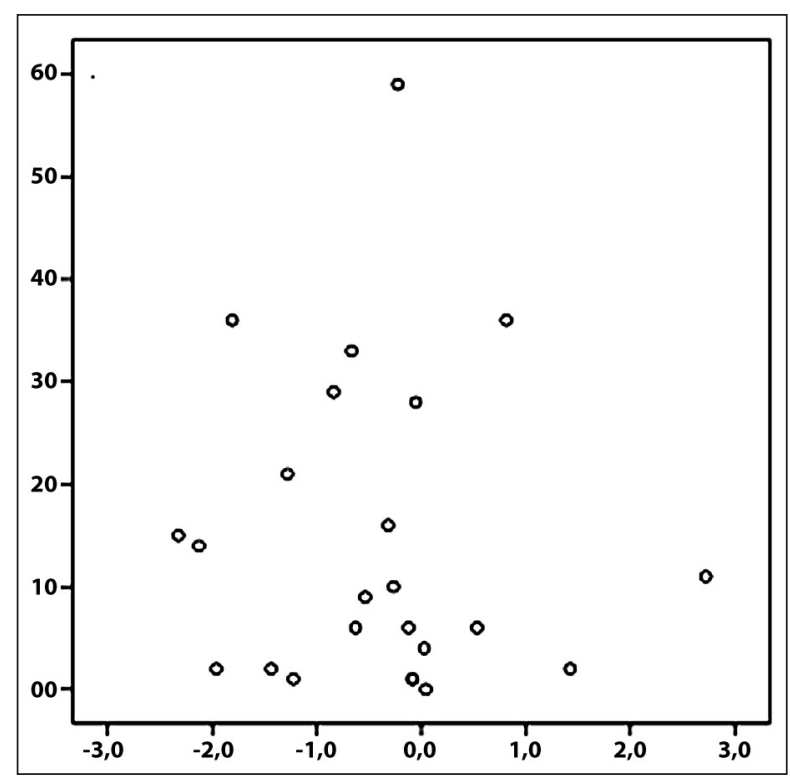

Figura 3 - Correlação entre hiperplasia neo-intimal e progressão de placa nos segmentos distais. Correlação de Spearman de $-0,166, p=0,36$.

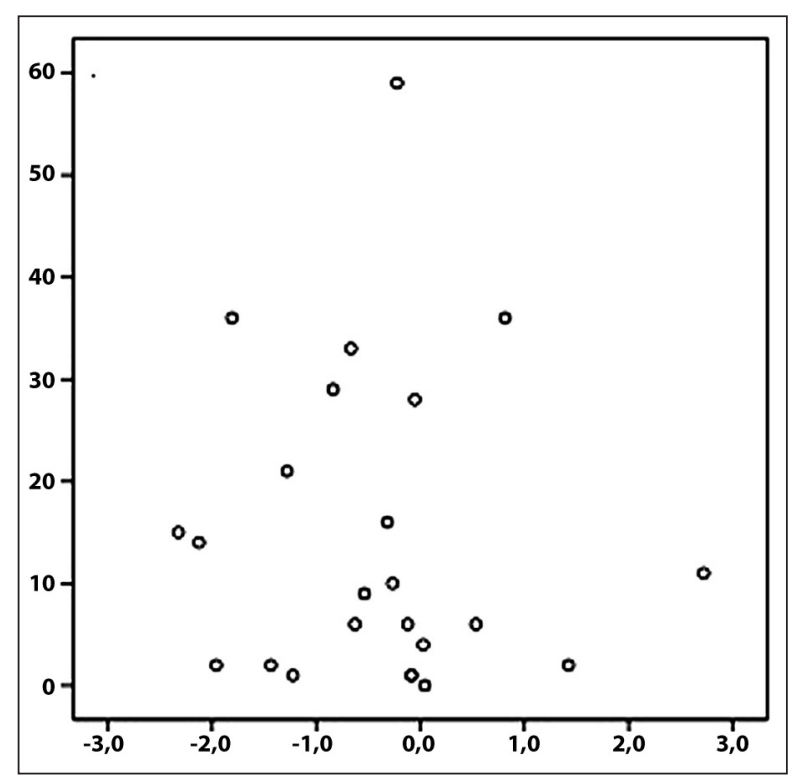

Figura 4 - Correlação entre hiperplasia neo-intimal e progressão de placa global (segmentos proximais e distais). Correlação de Spearman de $-0,026, p=0,89$.

ção ${ }^{12-16}$. O advento dos stents farmacológicos modificou esse cenário ${ }^{17,18}$. Por acrescentarem a sua composição um fármaco antiproliferativo e um polímero necessário para carreá-lo, a resposta inflamatória tornou-se mais complexa e variável de acordo com o tipo de fármaco e o polímero utilizados ${ }^{19,20}$. Ainda não está bem definida a duração desse processo na atual era, podendo variar conforme o tipo de stent farmacológico. 
Brito AFL, et al. Hiperplasia Neo-Intimal Intra-Stent e Progressão de Placa Aterosclerótica no Vaso-Alvo em Pacientes Tratados com Stents Liberadores de Zotarolimus. Rev Bras Cardiol Invas. 2008;16(4):450-455.

\section{Limitações}

Esta foi uma análise retrospectiva, com relativamente poucos pacientes. O perfil lipídico dessa coorte na ocasião do reestudo de seis meses não foi realizado de rotina, por não ter sido uma exigência do protocolo, ainda que todos os pacientes estivessem em uso de estatinas.

Ressalta-se que os achados deste estudo não podem ser extrapolados para populações de pacientes com perfis clínico-angiográficos diferentes, nem para os outros stents farmacológicos e não-farmacológicos, uma vez que o processo inflamatório secundário ao implante do stent parece estar relacionado a diversas variáveis, tais como tipo de metal utilizado e espessura de suas hastes, tipo de polímero utilizado, e tipo e dose do fármaco antiproliferativo empregado.

\section{CONCLUSÃO}

Nesta análise preliminar, a ausência de correlação entre a progressão de placa aterosclerótica fora do segmento tratado e o porcentual de obstrução intimal intra-stent do stent Endeavor ${ }^{\mathrm{TM}}$ aponta para mecanismos distintos envolvidos na gênese desses fenômenos.

\section{REFERÊNCIAS BIBLIOGRÁFICAS}

1. Hansson GK. Inflammation, atherosclerosis, and coronary artery disease. N Engl J Med. 2005;352(16):1685-95.

2. Libby P, Ridker PM, Maseri A. Inflammation and atherosclerosis. Circulation. 2002;105(9):1135-43.

3. Pai JK, Pischon T, Ma J, Manson JE, Hankinson SE, Joshipura $\mathrm{K}$, et al. Inflammatory markers and the risk of coronary heart disease in men and women. N Engl J Med. 2004;351(25): 2599-610.

4. Lasave LI, Abizaid AA, Paiva e Maia J, de Ribamar Costa J Jr., Feres F, Mattos LA, et al. Relationship between plasma C-reactive protein level and neointimal hyperplasia volume in patients with zotarolimus-eluting stents. Volumetric analysis by three-dimensional intracoronary ultrasound. Rev Esp Cardiol. 2007;60(9):923-31.

5. Bauriedel G, Skowasch D, Vaerst J, Jabs A, Andrié R, Lüderitz B. Association between progression of untreated coronary lesions and in-stent restenosis. Dtsch Med Wochenschr. $2002 ; 127(41): 2114-9$.

6. Jonas M, Fang JC, Wang JC, Giri S, Elian D, Har-Zahav Y, et al. In-stent restenosis and remote coronary lesion progression are coupled in cardiac transplant vasculopathy but not in native coronary artery disease. J Am Coll Cardiol. 2006; 48(3):453-61.
7. Mintz GS, Nissen SE, Anderson WD, Bailey SR, Erbel R, Fitzgerald PJ, et al. American College of Cardiology Clinical Expert Consensus Document on Standards for Acquisition, Measurement and Reporting of Intravascular Ultrasound Studies (IVUS). A report of the American College of Cardiology Task Force on Clinical Expert Consensus Documents. J Am Coll Cardiol. 2001;37(5):1478-92.

8. Nissen SE, Tuzcu EM, Schoenhagen P, Brown BG, Ganz P, Vogel RA, et al. Effect of intensive compared with moderate lipid-lowering therapy on progression of coronary atherosclerosis: a randomized controlled trial. JAMA. 2004;291(9): 1071-80.

9. Tardif JC, Grégoire J, L'Allier PL, Anderson TJ, Bertrand $\mathrm{O}$, Reeves $\mathrm{F}$, et al. Effects of the acyl coenzyme A: cholesterol acyltransferase inhibitor avasimibe on human atherosclerotic lesions. Circulation. 2004;110(21):3372-7.

10. Nissen SE, Nicholls SJ, Sipahi I, Libby P, Raichlen JS, Ballantyne $\mathrm{CM}$, et al. Effect of very high-intensity statin therapy on regression of coronary atherosclerosis: the ASTEROID trial. JAMA. 2006;295(13):1556-65.

11. Welt FG, Rogers C. Inflammation and restenosis in the stent era. Arterioscler Thromb Vasc Biol. 2002;22(11):1769-76.

12. Orford JL, Selwyn AP, Ganz P, Popma JJ, Rogers C. The comparative pathobiology of atherosclerosis and restenosis. Am J Cardiol. 2000;86(4B):6H-11H.

13. Kitamoto S, Egashira K, Takeshita A. Stress and vascular responses: anti-inflammatory therapeutic strategy against atherosclerosis and restenosis after coronary intervention. J Pharmacol Sci. 2003;91(3):192-6.

14. Farb A, Weber DK, Kolodgie FD, Burke AP, Virmani R. Morphological predictors of restenosis after coronary stenting in humans. Circulation. 2002;105(25):2974-80.

15. Yutani C, Ishibashi-Ueda H, Suzuki T, Kojima A. Histologic evidence of foreing body granulation tissue and de novo lesions in patients with coronary stent restenosis. Cardiology. 1999;92(3):171-7.

16. Inoue $T$, Kato $T$, Hikichi $Y$, Hashimoto $S$, Hirase $T$, Morooka $\mathrm{T}$, et al. Stent-induced neutrophil activation is associated with an oxidative burst in the inflammatory process, leading to neointimal thickening. Thromb Haemost. 2006;95(1):43-8.

17. Sousa JE, Serruys PW, Costa MA. New frontiers in cardiology: drug-eluting stents: Part I. Circulation. 2003;107(17): 2274-9.

18. Sousa JE, Serruys PW, Costa MA. New frontiers in cardiology: drug-eluting stents: Part II. Circulation. 2003;107(18): 2383-9.

19. Finn AV, Nakazawa G, Joner M, Kolodgie FD, Mont EK, Gold HK, et al. Vascular responses to drug eluting stents: importance of delayed healing. Arterioscler Thromb Vasc Biol. 2007;27(7):1500-10.

20. Caramori PR, Lima VC, Seidelin PH, Newton GE, Parker JD, Adelman AG. Long-term endothelial dysfunction after coronary artery stenting. J Am Coll Cardiol. 1999;34(6):1675-9. 